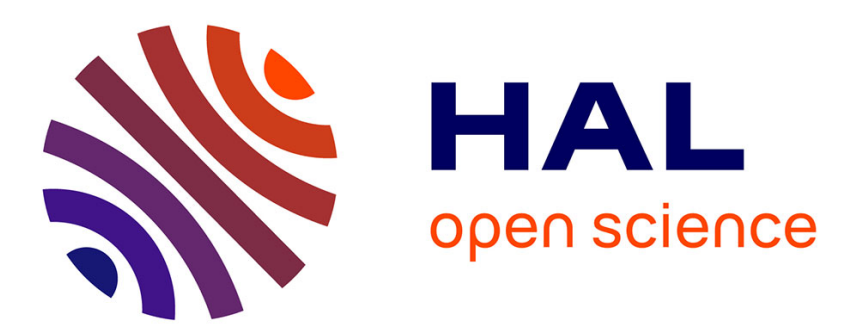

\title{
Results of bevacizumab as the primary treatment for retinal vein occlusions.
}

Marta S Figueroa, Inés Contreras, Susana Noval, Carolina Arruabarrena

\section{To cite this version:}

Marta S Figueroa, Inés Contreras, Susana Noval, Carolina Arruabarrena. Results of bevacizumab as the primary treatment for retinal vein occlusions.. British Journal of Ophthalmology, 2010, 94 (8), pp.1052. 10.1136/bjo.2009.173732 . hal-00561821

\section{HAL Id: hal-00561821 \\ https://hal.science/hal-00561821}

Submitted on 2 Feb 2011

HAL is a multi-disciplinary open access archive for the deposit and dissemination of scientific research documents, whether they are published or not. The documents may come from teaching and research institutions in France or abroad, or from public or private research centers.
L'archive ouverte pluridisciplinaire HAL, est destinée au dépôt et à la diffusion de documents scientifiques de niveau recherche, publiés ou non, émanant des établissements d'enseignement et de recherche français ou étrangers, des laboratoires publics ou privés. 


\section{TITLE PAGE}

Title: Results of bevacizumab as the primary treatment for retinal vein occlusions.

Authors: Marta S. Figueroa, MD, PhD $;{ }^{1,2}$ Inés Contreras, MD, PhD; ${ }^{1}$ Susana Noval, $\mathrm{MD}, \mathrm{PhD} ;{ }^{2,3}$ Carolina Arruabarrena, MD. ${ }^{2,4}$

${ }^{1}$ Hospital Universitario Ramón y Cajal. Madrid, Spain.

2. Vissum Corporación Oftalmológica. Madrid, Spain.

3. Hospital Universitario La Paz. Madrid, Spain.

${ }^{4 .}$ Hospital Príncipe de Asturias. Alcalá de Henares. Spain.

Corresponding author:

Inés Contreras

Address: c/ Ferraz n⿳355 2izquierda 28008 Madrid, Spain.

E-mail: inescon3@yahoo.com

Phone: +34630132955

Fax: +34913368126

No competing interests.

The Corresponding Author has the right to grant on behalf of all authors and does grant on behalf of all authors, an exclusive licence (or non-exclusive for government employees) on a worldwide basis to the BMJ Publishing Group Ltd and its Licensees to permit this article (if accepted) to be published in British Journal of Ophthalmology and 
any other BMJPGL products to exploit all subsidiary rights, as set out in our licence (http://group.bmj.com/products/journals/instructions-for-authors/licence-forms). 
ABSTRACT

Background: Our purpose was to evaluate the efficacy of intravitreal bevacizumab as the primary treatment of macular edema due to retinal vein occlusions.

Methods: Patients diagnosed with central retinal vein occlusion (CRVO) or branch retinal vein occlusion (BRVO) with visual acuity (VA) of less than 20/40 and macular edema with more than $300 \mu \mathrm{m}$ central retinal thickness were recruited. Patients that had received any prior treatment were excluded. After an initial intravitreal injection of bevacizumab, re-treatment was performed if intra- or subretinal fluid with distortion of the foveal depression was found in optical coherence tomography.

Results: 18 eyes with CRVO and 28 eyes with BRVO were included. During a 6-month period, the mean number of injections per patient was 3.7 (BRVO group) and 4.6 (CRVO group). In the BRVO group, mean baseline logMAR VA was 0.80 (SD 0.38) and macular thickness was $486.9 \mu \mathrm{m}$ (SD $138.5 \mu \mathrm{m})$. After six months, mean logMAR VA improved significantly to 0.44 (SD 0.34), $\mathrm{p}<0.001$. Mean macular thickness decreased significantly to $268.2 \mu \mathrm{m}$ (SD $62.5 \mu \mathrm{m}$ ), $\mathrm{p}<0.001$. In the CRVO group, mean baseline logMAR VA was 1.13 (SD 0.21) and macular thickness was $536.4 \mu \mathrm{m}$ (SD 107.1 $\mu \mathrm{m}$ ). Mean final logMAR VA improved significantly to 0.83 (SD 0.45), $\mathrm{p}<0.001$. Mean macular thickness decreased significantly to $326.17 \mu \mathrm{m}$ (SD $96.70 \mu \mathrm{m}$ ), $\mathrm{p}<0.001$.

Conclusions: Intravitreal bevacizumb seems to be an effective primary treatment option for macular edema due to retinal occlusions. Its main drawback is that multiple injections are necessary to maintain visual and anatomic improvements.

KEYWORDS: retinal vein occlusions, bevacizumab, macular edema, treatment, branch retinal vein occlusion, central retinal vein occlusion. 
Retinal vein occlusions are a frequent cause of visual impairment. Central retinal vein occlusion (CRVO) is less common than branch retinal vein occlusion (BRVO), but in both cases the development of macular edema (ME) is the main cause of visual loss. Treatment remains controversial. The Central Retinal Vein Occlusion Study Group found that laser treatment had a beneficial effect on neovasculatization but it failed to produce visual improvement in ME.[1] The Branch Vein Occlusion Study provided evidence that grid laser photocoagulation of the edematous macular area leads to a statistically significant benefit in terms of both visual acuity (VA) and persistence of ME as compared to the natural course of the disease.[2] However, mean improvement in VA was only 1.3 lines, and laser therapy leads to the development of scotomas.[2] Triamcinolone acetonide represents an alternative to laser treatment for retinal vein occlusions. Although results are positive in terms of reduction of ME and improvement in VA, its effects are only transient, requiring repeated injections.[3,4] Furthermore, its use has been associated with the development of posterior subcapsular cataracts and elevation of the intraocular pressure.[3-5] Initial treatment outcomes of a long-acting intravitreal fluocinolone acetonide sustained drug delivery implant have been reported in eyes with CRVO and chronic refractory ME.[6] Although VA improved in a significant proportion of eyes, with a reduction in ME, cataracts developed in all phakic patients in the study and 13 of 14 eyes required medical or surgical intraocular pressure lowering interventions.[6] Two clinical trials are ongoing to evaluate the treatment of ME in retinal vein occlusions with intravitreal corticosteroids. First, the multicenter randomized study SCORE, which is comparing the effectiveness and safety of standard care versus triamcinolone acetonide injection in patients with CRVO and BRVO. There is also a Phase 3 trial, evaluating the effect of an intravitreal sustained dexamethasone drug delivery system $\left(\right.$ Posurdex $^{\circledR}$ ) versus observation for ME secondary to retinal vein 
occlusions.[7] Different surgical approaches have also been reported for the treatment of both CRVO (vitrectomy with radial optic neurotomy) and BRVO (vitrectomy with or without sheathotomy at the arteriovenous crossing).[8]

Vascular endothelial growth factor (VEGF) is a cytokine produced by the hypoxic retina that increases vascular permeability, leading to ME. VEGF also stimulates endothelial cell hypertrophy, which reduces the capillary lumen and causes more ischemia and thus tends to perpetuate the edema. Anti-VEGF therapy could break this cycle and facilitate resolution of ME. Bevacizumab (Avastin; Genentech Inc., San Francisco, Calif.) is a monoclonal antibody that inhibits all isoforms of VEGF. It has been used off-label to treat several ischemic and edematous diseases. The purpose of this study is to evaluate the efficacy and safety of intravitreal bevacizumab as the sole treatment of retinal vein occlusions presenting with decreased VA due to ME.

Patients and methods

Prospective non-randomized interventional case series of patients diagnosed with CRVO or BRVO. Ethics committee approval was obtained. The experimental, offlabel use of bevacizumab was explained in detail to all patients before inclusion in the study and all patients granted informed consent.

At the visit prior to inclusion in the study, all patients underwent a complete ophthalmological examination, including best-corrected VA, biomicroscopy of the anterior and posterior segments, intraocular pressure measurement (Goldman applanation tonometry) and macular evaluation with optical coherence tomography, Fast Macular Thickness Map protocol, StratusOCT (Carl Zeiss Meditec Inc., Dublin, CA, USA). Central retinal thickness was measured with the Retinal Map analysis 
protocol in a circle of $1 \mathrm{~mm}$ in diameter centered on the fovea. OCT was repeated on each follow-up visit.

Inclusion criteria were as follows: VA of less than 20/40 and ME of more than $300 \mu \mathrm{m}$ of central retinal thickness in OCT. Patients previously treated for retinal vein occlusions or with any other ocular diseases were not included in the study.

Bevacizumab injections of $1.25 \mathrm{mg} / 0.05 \mathrm{ml}$ were administered under sterile conditions in the operating theatre. Antibiotic drops were prescribed for one week, starting three days before the injection.

Patient follow-up was performed one day, one week and one month after each injection and monthly thereafter. Eyes were retreated if either intra- or subretinal fluid with distortion of the normal foveal depression was found in the OCT examination.

Statistical analysis was performed with SPSS 13.0 software. VA was converted to $\log$ MAR prior to analysis. Quantitative variables are described with mean, median and range qualitative variables as percentages. The Shapiro-Wilk test was used to evaluate whether the variables analysed followed a normal distribution. Since variables did not follow a normal distribution, non-parametric tests were performed to evaluate changes in VA and retinal thickness.

\section{RESULTS}

Forty-six patients were included in the study, 18 patients with CRVO and 28 patients with BRVO. Twenty-one patients were women (46\%) and 25 men (54\%), with a mean age of 63.96 years (range between 50 and 78 years). No significant complications developed in either group after treatment with bevacizumab.

BRVO group 
In the BRVO group, mean baseline logMAR VA was 0.80 (SD 0.38; range 0.30 to 1.30); Snellen equivalents 0.22 (SD 0.16; range 0.05 to 0.5 ). Mean baseline macular thickness was $486.9 \mu \mathrm{m}$ (SD $138.5 \mu \mathrm{m}$; range 302 to $753 \mu \mathrm{m}$ ). The mean time elapsed between the diagnosis of BRVO and the first bevacizumab injection was 4 months (range from 5 days to 2 years).

Every patient was followed for at least 6 months after the first injection. During these 6 months, the mean number of injections per patient was 3.7 (range 1 to 6). Only one case resolved after a single injection.

Visual acuity improved progressively throughout the follow-up period (Figure 1, top), although the main improvement occurred during the first two months. This improvement was statistically significant as compared to baseline VA at all visits (Figure 1, top). At six months of follow-up, mean logMAR VA was 0.44 (SD 0.34; range 0 to 1.30); Snellen equivalent 0.46 ( $\mathrm{SD} 0.28$; range 0.05 to 1 ). Figure 2.A, shows the percentage of patients that gained 2 or more Snellen lines, that remained stable and that lost 2 or more lines. Only two patients lost 2 or more lines as compared to baseline during the first two months of treatment; however, after 3 months, vision improved or remained stable in all cases. The median improvement at 6 months was 2 Snellen lines. Final visual acuity was 0.5 or better (Snellen) in only $14 \%$ of patients with a baseline visual acuity equal to or lower than 0.2 , as compared with $100 \%$ of patients with a baseline VA higher than 0.2 .

Mean macular thickness decreased significantly after the injection of bevacizumab $(\mathrm{p}<$ 0.001; Wilcoxon test). The reduction persisted throughout the whole follow-up period (Figure 1, bottom). Final mean macular thickness was $268.2 \mu \mathrm{m}$ (SD $62.5 \mu \mathrm{m})$.

Pre-injection VA was significantly correlated with macular thickness before treatment $(\mathrm{p}<0.001, \mathrm{R}=0.686)$ and with final VA $(\mathrm{p}<0.001, \mathrm{R}=0.772)$. Similarly, final VA was 
significantly correlated with pre-injection macular thickness $(\mathrm{p}=0.001, \mathrm{R}=0.597)$ and with macular thickness at 6 months $(\mathrm{p}=0.001, \mathrm{R}=0.578)$.

\section{CRVO group}

In the CRVO group, mean baseline logMAR VA was 1.13 (SD 0.21, range 0.7 to 1.30 ); Snellen equivalent 0.08 (SD 0.05; range 0.05 to 0.2 ). Mean baseline macular thickness was $536.44 \mu \mathrm{m}$ (SD $107.14 \mu \mathrm{m}$; range 400 to $757 \mu \mathrm{m}$ ). The mean time elapsed between the diagnosis of CRVO and the first bevacizumab injection was 6 months (range 1 week to 2 years).

During the 6-month follow-up, the mean number of injections per patient was 4.6 (range 3 to 6). Mean final $\log$ MAR VA was 0.83 (SD 0.45; range 0.15 to 1.30 ); Snellen equivalent 0.23 (SD 0.21; range 0.05 to 0.7 ). Visual acuity improved progressively, although the main improvement occurred in the first month. This improvement was statistically significant compared to baseline VA at all visits (Figure 3, top). Median improvement at 6 months was 1 Snellen line.

Mean macular thickness decreased significantly after injections of bevacizumab $(\mathrm{p}<$ 0.001; Wilcoxon test). This reduction persisted throughout the follow-up period (Figure

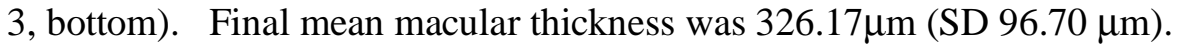

Pre-injection VA was significantly correlated with macular thickness before treatment $(\mathrm{p}=0.002, \mathrm{R}=0.679)$ and with final VA $(\mathrm{p}<0.001, \mathrm{R}=0.781)$. Similarly, final VA was significantly correlated with pre-injection macular thickness $(\mathrm{p}=0.07, \mathrm{R}=0.613)$ and with macular thickness at 6 months $(\mathrm{p}=0.044, \mathrm{R}=0.479)$. A correlation was also found between macular thickness reduction at one month and final VA: for each 100 microns of ME reduction, there was an improvement of 1 line in VA. 


\section{DISCUSSION}

Retinal vein occlusions cause visual loss due to initial hypoxia and delayed ME. The edema may cause an additional reduction in VA that often exceeds the primary ischemic damage; it is the most frequent complication of vein occlusions.[9] It has been shown that intravitreal levels of VEGF are significantly increased after retinal vein occlusions and that the degree of ME is correlated with VEGF levels in aqueous humor.[10] Therefore, several authors have studied the usefulness of intravitreal injections of bevacizumab in both types of retinal vein occlusions.[9]

Previous reports suggest that visual results are better in BRVO as compared with CRVO. The mean VA improvement was about $0.3 \log$ MAR units in most series of BRVO treated with bevacizumab.[11-14] An important feature of our study was that prior treatment was an exclusion criteria: patients were treated exclusively with bevacizumab. Few reports have prospectively studied patients with BRVO treated with bevacizumab as the sole treatment.

Russo et al evaluated bevacizumab versus macular grid laser photocoagulation for ME in 30 patients with BRVO: after treatment, eyes receiving bevacizumab had better VA than those receiving photocoagulation at all times.[13] VA improved by $0.3 \log$ MAR units 6 months after treatment with bevacizumab. Rensch et al evaluated the results of early treatment with bevacizumab after BRVO: mean VA improved by $0.21 \log$ MAR units 6 months after treatment. In this study, mean $\log$ MAR VA improved by 0.36 $\log$ MAR units after treatment. Thus, most studies to date report similarly positive VA results after the injection of bevacizumab. 
Most series have also shown a significant decrease in macular thickness after treatment with bevacizumab, though it is not always correlated with VA.[15,16] Rensch et al found that the improvement in VA correlated significantly with the decrease in macular thickness. We have found that both initial and final macular thicknesses are correlated with final VA. There may be several explanations for these results. Chung et al found that in the group of BRVO with no visual improvement, there was a higher proportion of angiographically documented macular ischemia. In this group, the decrease in central macular thickness was not accompanied by a visual acuity improvement. Although baseline visual acuity was not statistically different between groups, patients with no visual improvement had lower visual acuities than those that did improve. They also found that those patients that responded early to the first injections were more likely to benefit from the bevacizumab treatment.[17] Better initial visual acuities may reflect a preservation of macular function that may not be directly correlated with macular thickness; this would explain why patients with poor initial VA often have poor final VA in spite of the reduction of ME.

Visual results are much more variable in patients with CRVO, since no visual benefits, minimal improvements of 9 letters or significant VA improvements have been published.[11,12,17-20] In our study, mean VA improvement was 0.30 logMAR units, which is similar to that described by Rensch et al in a series of patients with ME caused by non-ischemic CRVO, with a mean duration prior to the first injection of 4.2 days (SD 3.6).[11] In our study, mean macular thickness in patients with CRVO decreased significantly after treatment. As in the patients with BRVO, macular thickness was correlated with VA. Rensch et al also found that the improvement in VA correlated significantly with the decrease in macular thickness.[11,21] Again, not all studies have found a relationship between retinal thickness and visual acuity in patients with CRVO. 
Beutel et al published a retrospective study with intravitreal bevacizumab for nonischemic CRVO in which they obtained a significant decrease in central retinal thickness without significant improvement of VA after 12 months of follow-up.[20] In a prospective study of ranibizumab for ME due to CRVO, VA and central retinal thickness were not correlated.[16] Sakamoto et al found that the preservation of the foveal inner/outer segment photoreceptor line after resolution of ME was significantly correlated with good visual function.[22] As in BRVO, initial VA may reflect macular function and thus bevacizumab may lead to poorer visual improvement in patients with CRVO as compared with patients with BRVO because the macular function may be more seriously damaged due to the greater size of the initial ischemia in CRVO.

No significant complications developed in our series after treatment with bevacizumab. The main drawback of bevacizumab for the treatment of $\mathrm{ME}$ after retinal vein occlusions is the need for repeated injections.[11,13,15-18,21-24] No retreatment schedule has been defined and new injections are performed at monthly or longer intervals at the discretion of the treating physician. However, Jaissle et al observed that the number of re-injections necessary to maintain the effect in BRVO declined over time.[9] Even though an optimal treatment regimen is as yet unclear, our data suggest that treatment should be initiated even if BRVO or CRVO have been present for some time: in our patients the temporal delay (range from 5 days to 2 years) from diagnosis to treatment initiation did not influence visual outcome, as reported in other series. An additional benefit that bevacizumab injections may provide as compared with other treatment options is that it may prevent the development of neovascularization or lead to its regression if it is already present.

In summary, this case series supports previous reports that suggest that bevacizumab is a valid, effective treatment for ME due to retinal vein occlusions. However, randomized 
multicenter studies are necessary to determine the optimal time after the vascular event for initiation of treatment and the schedule of injections. 


\section{FIGURE LEGENDS}

Figure 1. Branch retinal vein occlusion group. Top: Snellen visual acuity (VA) at baseline and at each visit. Bottom: Central macular thickness at baseline and at each visit. ( $\mathrm{p}$ values provided are those of the comparison between each visit and baseline values, Wilcoxon test).

Figure 2. Number of patients in which visual acuity remained stable, increased by two or more Snellen lines or decreased by two or more Snellen lines at each visit. A(top): Branch retinal vein occlusion (BRVO) group. B(bottom): Central retinal vein occlusion group (CRVO).

m months

Figure 3. Central retinal vein occlusion group. Top: Snellen visual acuity at baseline and at each visit. Bottom: Central macular thickness at baseline and at each visit. ( $\mathrm{p}$ values provided are those of the comparison between each visit and baseline values, Wilcoxon test). 


\section{REFERENCES}

1. Natural history and clinical management of central retinal vein occlusion. The Central Vein Occlusion Study Group. Arch Ophthalmol 1997;115:486-491.

2. Argon laser photocoagulation for macular edema in branch vein occlusion. The Branch Vein Occlusion Study Group. Am J Ophthalmol 1984;98:271-282.

3. Jonas JB, Akkoyun I, Kamppeter B, et al. Intravitreal triamcinolone acetonide for treatment of central retinal vein occlusion. Eur J Ophthalmol 2005;15:751758.

4. Jonas JB, Akkoyun I, Kamppeter B, et al. Branch retinal vein occlusion treated by intravitreal triamcinolone acetonide. Eye 2005;19:65-71.

5. Suarez-Figueroa M, Contreras I, Noval S. [Side-effects of triamcinolone in young patients]. Arch Soc Esp Oftalmol 2006;81:405-407.

6. Ramchandran RS, Fekrat S, Stinnett SS, et al. Fluocinolone acetonide sustained drug delivery device for chronic central retinal vein occlusion: 12-month results. Am J Ophthalmol 2008;146:285-291.

7. Kuppermann BD, Blumenkranz MS, Haller JA, et al. Randomized controlled study of an intravitreous dexamethasone drug delivery system in patients with persistent macular edema. Arch Ophthalmol 2007;125:309-317.

8. Rehak J, Rehak M. Branch retinal vein occlusion: pathogenesis, visual prognosis, and treatment modalities. Curr Eye Res 2008;33:111-131.

9. Abegg M, Tappeiner C, Wolf-Schnurrbusch U, et al. Treatment of branch retinal vein occlusion induced macular edema with bevacizumab. BMC Ophthalmol 2008;8:18. 
10. Funk M, Kriechbaum K, Prager F, et al. Intraocular concentrations of growth factors and cytokines in retinal vein occlusion and the effect of therapy with bevacizumab. Invest Ophthalmol Vis Sci 2009;50:1025-1032.

11. Rensch F, Jonas JB, Spandau UH. Early intravitreal bevacizumab for nonischaemic branch retinal vein occlusion. Ophthalmologica 2009;223:124-127.

12. Jaissle GB, Leitritz M, Gelisken F, et al. One-year results after intravitreal bevacizumab therapy for macular edema secondary to branch retinal vein occlusion. Graefes Arch Clin Exp Ophthalmol 2009;247:27-33.

13. Russo V, Barone A, Conte E, et al. Bevacizumab compared with macular laser grid photocoagulation for cystoid macular edema in branch retinal vein occlusion. Retina 2009;29:511-515.

14. Kreutzer TC, Alge CS, Wolf AH, et al. Intravitreal bevacizumab for the treatment of macular oedema secondary to branch retinal vein occlusion. $\mathrm{Br} J$ Ophthalmol 2008;92:351-355.

15. Chung EJ, Hong YT, Lee SC, et al. Prognostic factors for visual outcome after intravitreal bevacizumab for macular edema due to branch retinal vein occlusion. Graefes Arch Clin Exp Ophthalmol 2008;246:1241-1247.

16. Spaide RF, Chang LK, Klancnik JM, et al. Prospective study of intravitreal ranibizumab as a treatment for decreased visual acuity secondary to central retinal vein occlusion. Am J Ophthalmol 2009;147:298-306.

17. Beutel J, Ziemssen F, Luke M, et al. Intravitreal bevacizumab treatment of macular edema in central retinal vein occlusion: one-year results. Int Ophthalmol 2008 Dec 20. [Epub ahead of print] 
18. Prager F, Michels S, Kriechbaum K, et al. Intravitreal bevacizumab (Avastin) for macular oedema secondary to retinal vein occlusion: 12-month results of a prospective clinical trial. Br J Ophthalmol 2009;93:452-456.

19. Iturralde D, Spaide RF, Meyerle CB, et al. Intravitreal bevacizumab (Avastin) treatment of macular edema in central retinal vein occlusion: a short-term study. Retina 2006;26:279-284.

20. Ferrara DC, Koizumi H, Spaide RF. Early bevacizumab treatment of central retinal vein occlusion. Am J Ophthalmol 2007;144:864-871.

21. Rensch F, Jonas JB, Spandau UH. Early intravitreal bevacizumab for nonischaemic central retinal vein occlusion. Acta Ophthalmol 2009;87:77-81.

22. Sakamoto A, Tsujikawa A, Ota M, et al. Evaluation of potential visual acuity in eyes with macular oedema secondary to retinal vein occlusion. Clin Experiment Ophthalmol 2009;37:208-216.

23. Gregori NZ, Gaitan J, Rosenfeld PJ, et al. Long-term safety and efficacy of intravitreal bevacizumab (Avastin) for the management of central retinal vein occlusion. Retina 2008;28:1325-1337.

24. Wu L, Arevalo JF, Roca JA, et al. Comparison of two doses of intravitreal bevacizumab (Avastin) for treatment of macular edema secondary to branch retinal vein occlusion: results from the Pan-American Collaborative Retina Study Group at 6 months of follow-up. Retina 2008;28:212-219. 

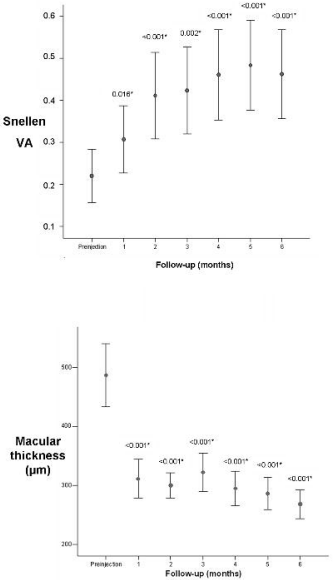

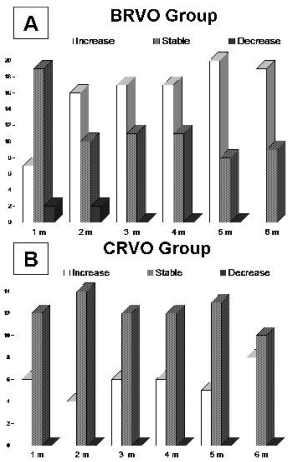


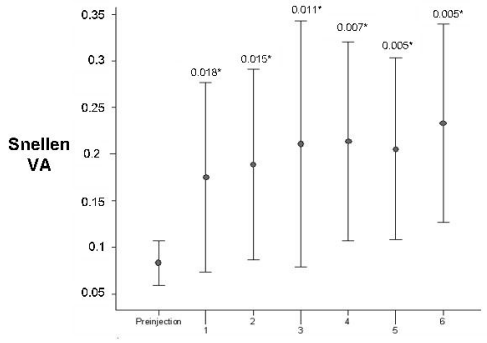

Follow-up (months)

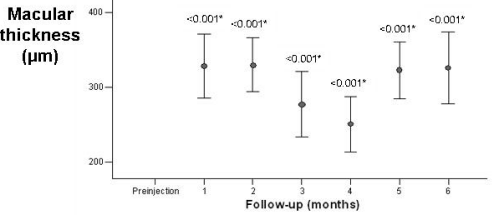

